\title{
Bonding strategies to full-contour zirconia: Zirconia pretreatment with piranha solution, glaze and airborne-particle abrasion
}

\author{
Sabrina A. Feitosa ${ }^{\mathrm{a}, \mathrm{b}}$, Nelson B. Lima ${ }^{\mathrm{c}}$, Walter K. Yoshito ${ }^{\mathrm{c}}$, Fernanda Campos ${ }^{\mathrm{b}}$, Marco A. Bottino ${ }^{\mathrm{b}}$, \\ Luiz F. Valandro ${ }^{\text {, }}$, Marco C. Bottino ${ }^{\text {a,* }}$ \\ a Department of Biomedical and Applied Sciences, Division of Dental Biomaterials, Indiana University School of Dentistry, Indianapolis, IN, USA \\ ${ }^{\mathrm{b}}$ Department of Dental Materials and Prosthodontics, Institute of Science and Technology, Universidade Estadual Paulista-UNESP, São José dos Campos, São Paulo, Brazil \\ ${ }^{\mathrm{c}}$ Materials Science and Technology Center, Institute for Energy and Nuclear Research, IPEN, São Paulo, São Paulo, Brazil \\ d Department of Restorative Dentistry, Division of Prosthodontics, Federal University of Santa Maria, Santa Maria, Rio Grande do Sul, Brazil
}

\section{A R T I C L E I N F O}

\section{Keywords:}

Glaze

Zirconia

Scratch test

Surface treatment

Shear test

Piranha solution

\begin{abstract}
A B S T R A C T
This study aimed to evaluate the effect of various zirconia surface pretreatments on the adhesion between full contour 3Y-TZP zirconia and glaze, and the shear bond strength (SBS) between glazed/3Y-TZP and resin cement. Specimens were allocated into groups: GL-glaze; $\mathrm{AL}+\mathrm{GL}$-sandblasting with $\mathrm{Al}_{2} \mathrm{O}_{3}+\mathrm{GL}$; CJ + GL-tribochemical silica coating $\left(\right.$ Cojet $\left.^{\circledast} / \mathrm{CJ}\right)+\mathrm{GL}$; PS + GL-piranha solution + GL; and CJ. Adhesion between 3Y-TZP and GL was evaluated using the scratch test. Surface topography and glaze thickness were evaluated by using a scanning electron microscope (SEM). For SBS, glazed/3Y-TZP surface was etched with hydrofluoric acid and a silane was applied. For CJ only the silane was applied. Samples were tested after $24 \mathrm{~h}$ (24 h wet) or after 15,000 thermal cycles and 90 days storage (thermocycled). After SBS, the type of failure was classified as: adhesive, mixed or cohesive. The data were analyzed using two-way ANOVA and Tukey's test. SEM analysis after scratch test revealed circular cracks in the GL group and conformal cracks in the others groups. SEM micrographs suggested that zirconia specimens submitted to airborne-particle abrasion presents rougher and porous surface when compared to surfaces treated with GL and PS. The glaze layer was approximately $1.86 \mu \mathrm{m}$ thick in all groups. After $24 \mathrm{~h}$, SBS test showed highest values for AL + GL and CJ + GL and were significantly higher when compared to the GL group. Differences were not significant between PS + GL and the other groups. After aging (thermocycling + storage), groups GL and CJ presented no statistically significant difference compared to $24 \mathrm{~h}$ and aged $\mathrm{AL}+\mathrm{GL}, \mathrm{CJ}+\mathrm{GL}$ and PS + GL groups. The predominant type of failure was mixed. 3Y-TZP surface treatment with glaze application could be considered as an alternative treatment, since it yielded a similar resin bond strength without the need for airborne-particle abrasion.
\end{abstract}

\section{Introduction}

Zirconia is widely used as a dental restorative material due to its proven biocompatibility and superior mechanical properties. In clinical dentistry, to achieve reliable and durable bond strength between silicabased ceramics and resin cements, the use of hydrofluoric acid (HF) followed by the application of a silane coupling agent are currently widely accepted as important steps [1-3]. Among acids, HF is the most commonly used and has been shown to improve the bonding ability between the glassy matrix of silica-based ceramics and resin cement $[2,4-6]$. HF reacts with the ceramic glass matrix by forming hexafluorosilicates $[5,7]$ thus effectively removing the matrix to expose the crystalline structure and create a rough and retentive surface that increases the mechanical interlocking with the resin cement $[5,7]$. The next step is the use of a silane coupling agent such as methacryloxypropyl trimethoxysilane (MPS) to improve the chemical bonding between ceramic and resin cement. Briefly, silane molecules react with water resulting in silanol groups (-Si-OH) and form the methoxy groups $\left(-\mathrm{Si}-\mathrm{O}-\mathrm{CH}_{3}\right)$. The silanol group reacts with the silica present on the ceramic surface by forming a siloxane network (-Si-O-Si-O-) [8-10]. However, this micromechanical/chemical bonding mechanism is not effective on 3Y-TZP. This is because HF is unable to successfully erode the highly crystalline surface of these ceramics and therefore does not create the desired microretentive surface topography [11-13]. Thus, discovering the best way to improve bonding and durability of zirconia restorations has been a common goal of researchers.

\footnotetext{
* Correspondence to: Indiana University School of Dentistry, Department of Biomedical and Applied Sciences, Division of Dental Biomaterials, 1121 W. Michigan Street, Indianapolis, IN 46202, USA.

E-mail address: mbottino@iu.edu (M.C. Bottino).
} 
Table 1

Full-contour zirconia surface treatments applied for each group.

\begin{tabular}{|c|c|}
\hline Groups & Surface treatment \\
\hline GL & A low-fusing porcelain glaze was applied onto the ceramic surface with a brush, dried at $37^{\circ} \mathrm{C}$ for $2 \mathrm{~h}$, and sintered following the manufacturer's instructions. \\
\hline $\mathbf{A L}+\mathbf{G L}$ & $\begin{array}{l}\text { Zirconia specimens were air-abraded with } 50 \mu \mathrm{m} \text { aluminum oxide particles }\left(\mathrm{Al}_{2} \mathrm{O}_{3}\right) \text { (Batch \#3150313, Patterson Dental Supply, Inc., Saint Paul, MN, USA) for } 30 \mathrm{~s} \text {, } \\
\text { under } 2.8 \text { bars and from a distance of approximately } 10 \mathrm{~mm} \text {. A low-fusing porcelain glaze was applied onto the ceramic surface with a brush, dried at } 37^{\circ} \mathrm{C} \text { for } 2 \mathrm{~h} \text {, and } \\
\text { sintered following the manufacturer's instructions. }\end{array}$ \\
\hline $\mathbf{C J}+\mathbf{G L}$ & $\begin{array}{l}\text { Zirconia specimens were silica-coated using particle abrasion with } 30 \mu \mathrm{m} \text { silica-coated aluminum oxide particles (Cojet }{ }^{\circ} \text {-Sand, } 3 \mathrm{M} \text { ESPE AG, Seefeld, Germany; Lot \# } \\
501661 \text { ) for } 30 \mathrm{~s} \text {, under } 2.8 \text { bars and from a distance of approximately } 10 \mathrm{~mm} \text {. A low-fusing porcelain glaze was applied onto the ceramic surface with a brush, dried at } \\
37^{\circ} \mathrm{C} \text { for } 2 \mathrm{~h} \text {, and sintered following the manufacturer's instructions. }\end{array}$ \\
\hline $\mathbf{P S}+\mathbf{G L}$ & $\begin{array}{l}\text { Zirconia specimens were chemically pretreated with Piranha Solution } 3: 1 \text { - Sulfuric acid }\left(\mathrm{H}_{2} \mathrm{SO}_{4}\right) / 30 \% \mathrm{H}_{2} \mathrm{O}_{2} \text {. Zirconia samples were immersed for } 30 \text { min in the } \\
\text { solution, rinsed with distilled water for } 5 \mathrm{~min} \text {, and immersed in distilled water for } 20 \mathrm{~min} \text {. A low-fusing porcelain glaze was applied to the ceramic surface with a brush, } \\
\text { dried at } 37^{\circ} \mathrm{C} \text { for } 2 \mathrm{~h} \text {, and sintered following the manufacturer's instructions. }\end{array}$ \\
\hline CJ & $\begin{array}{l}\text { Zirconia specimens were air-abraded with } 30 \mu \mathrm{m} \text { silica-coated aluminum oxide particles (Cojet) (Cojet }{ }^{\oplus} \text {-Sand, 3M ESPE AG, Seefeld, Germany; Lot \# } 501661 \text { ) for } 30 \mathrm{~s} \text {, } \\
\text { under } 2.8 \text { bars and from a distance of approximately } 10 \mathrm{~mm} \text {. }\end{array}$ \\
\hline
\end{tabular}

Zirconia surface treatments can be divided into "traditional" and "new" [14] with traditional treatments including the use of restorative materials containing MDP (phosphate ester monomers), airborne-particle abrasion, tribochemical silica coating (Rocatec or Cojet, 3M ESPE) and zirconia primers [14]. On the other hand, "new treatments" include the use of selective infiltration etching [15-17], glaze-on technique, hot etching solution and chemical treatments (piranha solution (PS)-a mixture of 3:1 sulfuric acid:30\% hydrogen peroxide and up to $40 \% \mathrm{HF}$ ) [14].

Airborne-particle abrasion has been shown to improve the bonding ability between 3Y-TZP and resin cements and the glaze-on technique has been suggested as an alternative for airborne-particle abrasion treatment due to potential ceramic weakening effects associated with zirconia tetragonal to monoclinic $(\mathrm{t} \rightarrow \mathrm{m})$ phase transformation. The glaze-on technique with multi-phase glaze containing a major lithium disilicate phase results in a zirconia surface amenable to etching and adhesive bonding and therefore can be an alternative treatment for zirconia [18]. A similar method described as "internal coating technique" uses a silica-based ceramic on the zirconia surface followed by silane application to improve bond strength [19]. In addition, some studies have reported using a chemical treatment with a PS [20,21], which is known as a cleaning reagent and a strong oxidizing solution that hydroxylates most surfaces and makes them hydrophilic thus improving their hydroxylation and bond strength to adhesive monomers $[14,20,21]$.

The durability of a glaze-coating technique could be influenced by its adhesion to the substrate and depends on the properties of the substrate and the coating $[22,23]$. There is a lack of information regarding the bonding ability between 3Y-TZP and glaze, which can affect long-term stability of the technique. One way to evaluate glaze-zirconia bonding is by using the scratch test, which determines the cohesive and adhesive properties of thin films during coat detachment $[22,23]$. The test is conducted by applying a progressive or constant force on the substrate through a diamond stylus at constant speed so as to gently damage/scratch the coat [24].

In order to avoid problems related to chipping of the veneering ceramic and limited occlusal and palatal space, full-contour zirconia (FCZ) without the veneering ceramic has been developed [25-28]. In clinical practice, dental restoration treatments using FCZ can be improved by adequate treatment of the zirconia surface. Therefore, the aims of the present study were to evaluate the effect of different zirconia pretreatments on 1) the adhesion between 3Y-TZP and glaze, and 2) the SBS between glazed/3Y-TZP and resin cement. Two hypotheses were tested in this study: 1) zirconia pretreatment via airborne-particle abrasion, tribochemical silica coating and PS improves the glazecoating adhesion to the FCZ and to the resin cement; 2) After aging (i.e., thermocycling and water storage) the zirconia surface pretreatment combined with a glaze application would provide better results of shear bond strength when compared to the single treatments (CJ and GL).

\section{Materials and methods}

\subsection{Preparation of FCZ specimens}

One hundred and fifty FCZ ceramic bars (3Y-TZP, Lot \#P02286, Diazir ${ }^{\circ}$, Ivoclar-Vivadent, Amherst, NY, USA) were cut into blocks (10 $\times 10 \times 3 \mathrm{~mm}^{3}$ ) using a diamond-wafering blade mounted on a precision saw machine (ISOMET 1000, Buehler Ltd., Lake Bluff, IL, USA). The ceramic blocks were sintered at $1500{ }^{\circ} \mathrm{C}$ for $2 \mathrm{~h}$ in a high-temperature furnace (Lindberg/Blue M, Kendro Laboratory Products, Inc., Asheville, NC, USA) according to the manufacturer's instructions. The ceramic blocks were then finished with SiC paper (LECO Corporation, St. Joseph, MI, USA), cleaned in an ultrasonic bath containing isopropyl alcohol for $5 \mathrm{~min}$, rinsed with water, air-dried, and randomly distributed into five groups (Table 1 ).

\subsection{Scanning electron microscopy (SEM)}

Scanning electron microscopy (SEM, JSM-6390, JEOL, Tokyo, Japan) was used to analyze the ceramic surface morphology after each conditioning method (Table 1). The specimens were mounted onto a metallic stub and submitted to sputter coating with gold.

\subsection{Scratch test}

The bonding ability between FCZ and glaze $(\mathrm{N}=3$; except for the CJ group - without glaze application) was assessed by the scratch test (ASTM C1624-05) [24] using a $200 \mu \mathrm{m}$ spherical Rockwell C diamond stylus with a progressive vertical load that increased linearly from 0 to $30 \mathrm{~N}$ at a constant speed. After the test, the critical damage was viewed through the SEM and the images were qualitatively analyzed.

\subsection{Glaze thickness}

Glaze-coated FCZ ceramic bars were vertically mounted in epoxy resin after applying the glaze. The zirconia/glaze interface was evaluated through SEM and the glaze thickness was measured using Image $\mathrm{J}$ software (National Institutes of Health, Bethesda, MD, USA).

\subsection{Shear bond strength test and failure analysis}

The glaze-modified FCZ surface was etched with 5\% HF (Lot \#R53559, Ivoclar Vivadent) for $90 \mathrm{~s}$, rinsed for $60 \mathrm{~s}$ and air-dried. A silane agent (Lot \#R50513, Monobond Plus, Ivoclar-Vivadent) was then applied with a brush and was left undisturbed for $1 \mathrm{~min}$, and the solvent was air-dried. Resin cement buttons $(\sim 2.2-\mathrm{mm}$ in height and $2.38-\mathrm{mm}$ in diameter) were fabricated using a specially fabricated jig (Ultradent Products, Inc., South Jordan, UT, USA) and a cylindrical Teflon mold; they were then placed over each zirconia specimen. Resin cement (Lot \#R25959, Multilink ${ }^{\circledR}$ Implant, Ivoclar-Vivadent) was applied to the 


\section{(A)}
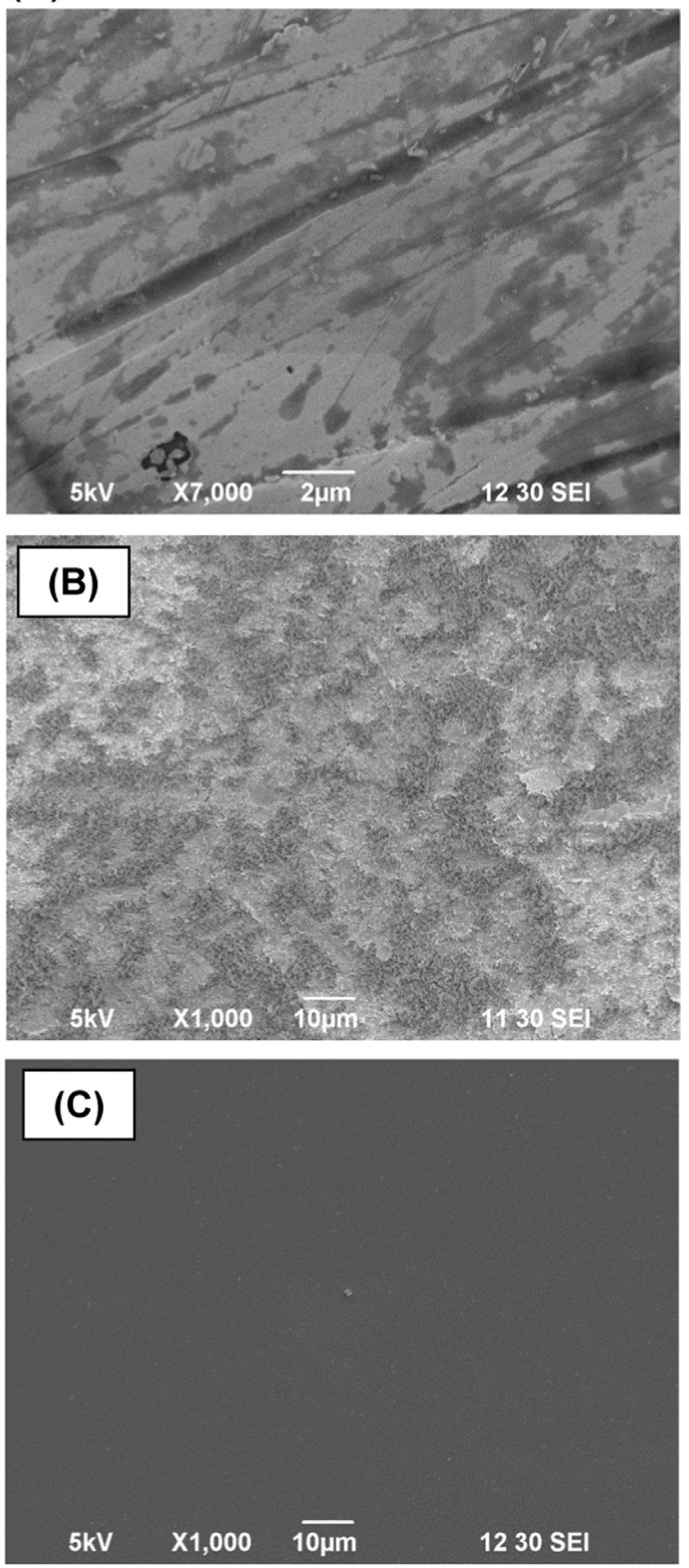

Fig. 1. Representative SEM micrographs of the FCZ ceramic surface after being subjected to (A) piranha solution $\left(\mathrm{H}_{2} \mathrm{SO}_{4} / 30 \% \mathrm{H}_{2} \mathrm{O}_{2}=3: 1\right)$, (B) airborne-particle abrasion with Cojet $^{\circ}$ (3M ESPE), and (C) glaze application.

mold and it was light-cured (Demi L.E.D. Dental Curing Light, Kerr Corporation, Middleton, WI, USA) following the manufacturer's instructions. Intensity of the light $\left(\sim 1200 \mathrm{~mW} / \mathrm{cm}^{2}\right)$ was checked with a radiometer (Cure Rite, Curing Light Meter, Dentsply Caulk, Division of Dentsply International Inc., Milford, DE, USA) prior to bonding. Specimens from each group were assigned to subgroups as follows: 1) $24 \mathrm{~h}$ (wet, no aging): the specimens were kept in water for $24 \mathrm{~h}$ at $37^{\circ} \mathrm{C}$ before testing; 2) Aging (thermocycled): the specimens were thermocycled $\left(15,000\right.$ cycles, $8-48{ }^{\circ} \mathrm{C}$, dwell time of $30 \mathrm{~s}$, transfer time of $\left.10 \mathrm{~s}\right)$ [29], and water storage: the specimens were kept in water at $37^{\circ} \mathrm{C}$ for $90 \mathrm{~d}$ before testing. In addition, the water was changed every month after thermocycling. The shear bond strength was evaluated using a dedicated jig attached to a Universal Testing Machine (ElectroPuls

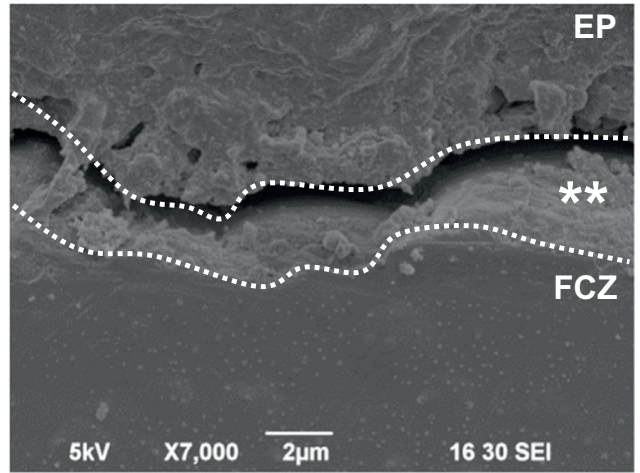

Fig. 2. Representative SEM micrograph of the glaze-coated FCZ ceramic. The ceramic sample was vertically mounted in epoxy resin after the glaze treatment, as a result, the images shows the zirconia surface (FCZ), glaze layer $\left(^{* *}\right)$ and the epoxy resin (EP) $(\times 7000)$.

E3000, Instron, Norwood, MA, USA). The load was applied to the adhesive interface until failure $(1 \mathrm{~mm} / \mathrm{min})$. The maximum stress to produce fracture was recorded $\left(\mathrm{N} / \mathrm{mm}^{2}=\mathrm{MPa}\right)$.

The fractured surfaces on the FCZ specimens was examined under light microscopy, and the mode of failure was classified as: Cohesive of the resin cement, Cohesive-ceramic, Adhesive, and Mixed [11]. Additionally, after gold-sputter coating, representative specimens from each group were imaged at different magnifications under SEM.

\subsection{Data analysis}

SBS data were analyzed using two-way ANOVA and Tukey's test ( $\alpha$ $=0.05$ ) to examine the effects of group and condition on shear bond strength.

\section{Results}

\subsection{SEM}

The SEM micrographs revealed scratches on the FCZ surface (Fig. 1) without superficial defects and gaps that could have resulted from the polishing protocol. In all groups (GL; $A L+G L ; C J+G L$; and $S P+G L$ ), the specimens treated with glaze presented a smooth topography (SEM). From the topography (Fig. 1), it was evident that airborneparticle abrasion $\left(\mathrm{Al}_{2} \mathrm{O}_{3}\right.$ and $\left.\mathrm{SiO}_{2}\right)$ presented a rougher surface when compared to chemical treatment with PS.

\subsection{Glaze thickness}

The glaze layer was approximately $1.86 \pm 0.05 \mu \mathrm{m}$ thick in all the glazed groups (Fig. 2).

\subsection{Scratch test}

The scratch test pattern (qualitative analysis) showed that the groups, AL + GL, CJ + GL, and GL + PS presented similar types of failure, classified as conformal cracks, which represents a cohesive failure of the glaze coating (Fig. 3).

\subsection{Shear bond strength test and failure analysis}

Two-way ANOVA showed that the zirconia surface treatment and storage significantly affected the bond strength between Y-TZP and resin cement (Table 2).

For $24 \mathrm{~h}$ (immediate condition), $\mathrm{AL}+\mathrm{GL}$ and $\mathrm{CJ}+\mathrm{GL}$ presented the statistically highest means, followed by PS + GL (Table 3). After storage, the differences between groups were not statistically significant (Table 3). When the effect of storage was compared between each 

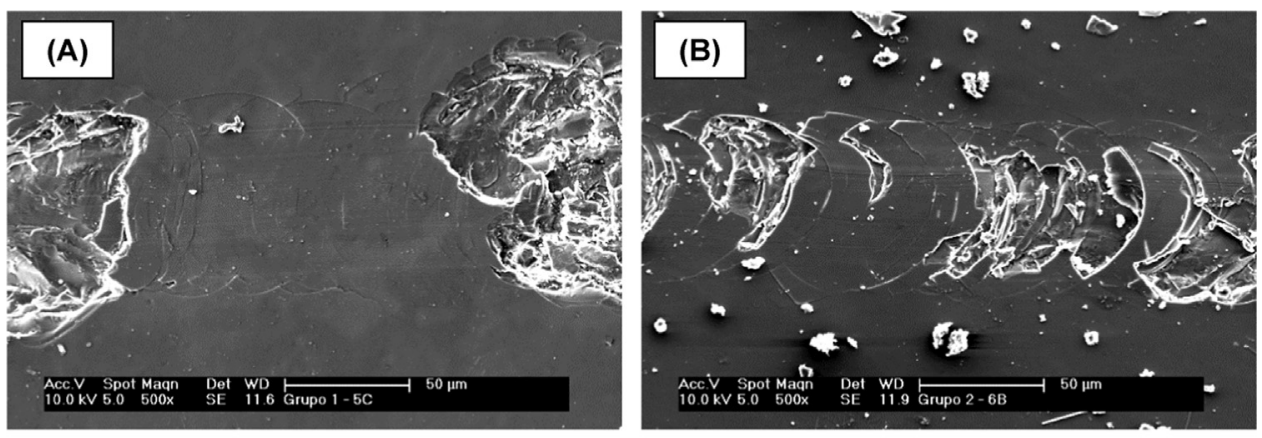

Fig. 3. Representative SEM micrographs of the glazecoated zirconia ceramic samples after the scratch test. (A) GL; (B) $\mathrm{AL}+\mathrm{GL}$; (C) $\mathrm{CJ}+\mathrm{GL}$; and (D) PS + GL. Groups $(B \rightarrow$ D) presented similar scratch pattern.
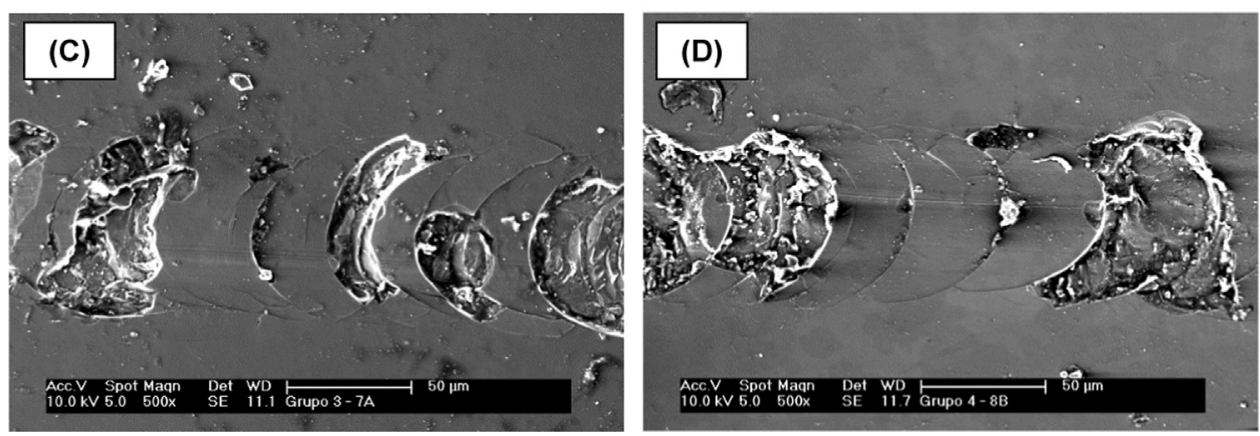

Table 2

Analysis of variance of the shear bond strength data (MPa).

\begin{tabular}{llllll}
\hline Source & DF & SS & MS & F & P \\
\hline Group & 4 & 1250.36 & 312.59 & 6.30 & 0.0001 \\
Condic & 1 & 3819.24 & 3819.24 & 76.93 & 0.0001 \\
Group*Condition** & 4 & 649.13 & 162.28 & 3.27 & 0.0139 \\
Error & 119 & 5907.74 & 49.64 & & \\
Total & 128 & & & & \\
\hline
\end{tabular}

Table 3

Means (standard deviation) of bond data (in MPa) and All-Pairwise Comparisons Tukey Test, considering the surface treatments and storage conditions.

\begin{tabular}{llllll}
\hline \multirow{2}{*}{ Storage } & \multicolumn{5}{l}{ Surface treatments } \\
\cline { 2 - 6 } & GL & AL + GL & CJ + GL & PS + GL & CJ \\
\hline \multirow{2}{*}{$\mathbf{2 4 h}$} & $18.8(8.7)$ & $27.8(5.0) \mathrm{A}$ & $29.7(9.4)$ & $26.3(6.29)$ & $18.2(6.1)$ \\
& BC & & A & AB & BCD \\
Storage & $14.3(7.1)$ & $11.09(3.8) \mathrm{D}$ & $\begin{array}{l}16.4(9.4) \\
\text { CD }\end{array}$ & $\begin{array}{l}15.9(3.65) \\
\text { CD }\end{array}$ & $\begin{array}{l}11.2(5.4) \\
\text { CD }\end{array}$ \\
& CD & & CD & CD & \\
\hline
\end{tabular}

Means and standard deviations (S.D.) in MPa.

Uppercase different letter imply significant difference among group within condition $(\mathrm{p}<0.05)$.

treatment, it became evident that the bonds in the AL + GL, CJ + GL, and PS + GL groups reduced significantly after aging (Table 3). Mixed failures were predominant in all the tested groups. Adhesive failures were also observed in groups GL, AL + GL, and PS + GL (Figs. 4 and 5).

\section{Discussion}

In the present study, we first investigated whether pretreatment of zirconia can improve adhesion of the glaze-coating to zirconia and the resin cement. According to the collected data, our hypothesis that zirconia pretreatment via airborne-particle abrasion, tribochemical silica coating and PS improves the glaze-coating adhesion to the FCZ and to the resin cement is accepted. Second, we analyzed whether a combination of treatments (AL + GL, CJ + GL, PS + GL) would improve SBS when compared to the single treatments ( $\mathrm{CJ}$ and $\mathrm{GL}$ ). The GL treatment (alone or combined) was suggested in order to allow the use of the conventional bond strategy for glass-ceramics using HF and silane. Based on the results our second hypothesis that aging via thermocycling and water storage of the pretreated and glaze applied zirconia surface improves SBS compared to the single treatments (CJ and GL) is rejected.

The first surface treatment used in this present study was using PS. The rationale to test PS in this study was to avoid the impact of airborne-particle abrasion on zirconia. Previous studies compared the effect of different chemical treatments in combination with airborneparticle abrasion [20,21]. They found that the use of PS for 4 days in combination with silane increased the adhesive potential although tribochemical silica coating was the most effective treatment [20]. Another study that investigated the effectiveness of PS in bonding to zirconia found significantly lower SBS in the groups treated with PS when compared with the group submitted to airborne-particle abrasion combined with zirconia primer [21].

Unlike the above two studies $[20,21]$, in the present study, we incubated zirconia specimens in PS for a short time of $30 \mathrm{~min}$, and cleaned with distilled water for $5 \mathrm{~min}$ and kept them in distilled water for another $20 \mathrm{~min}$. Although, distinct PS and treatment regimens have been used, no consensus has been reached on the ideal protocol for the use of PS in zirconia bonding $[20,21]$. Nonetheless, it is clear that regardless of the exposure time (4 days vs. $30 \mathrm{~min}$ ), none of the studies led to higher values of bond strength (compared to other groups) when PS treatment was employed. The SEM findings also corroborate with this conclusion, since no enhancement on the surface roughness could be seen.

The second surface pretreatment evaluated in this study was the airborne-particle abrasion with aluminum oxide or tribochemical silica coating. These treatments are considered gold standard to improve the bonding between zirconia and resin cements [12]. SEM micrographs (Fig. 1) after treatments showed a rough zirconia surface with porosities and undercuts. This pattern resulted in a ceramic with increased surface area, favorable to interlocking with the resin cement. In addition, the tribochemical silica coating (CJ) provide a silica-coated zirconia surface, which is known to be favorable for interaction with the silane agent and resin cement [12].

After the surface pretreatments, glaze was applied on the zirconia in the $\mathrm{AL}+\mathrm{GL}, \mathrm{CJ}+\mathrm{GL}$ and PS + GL groups to achieve a thin layer of glaze since a previous study reported marginal fit discrepancies when working with overglazed zirconia restorations [30]. Analysis of the 

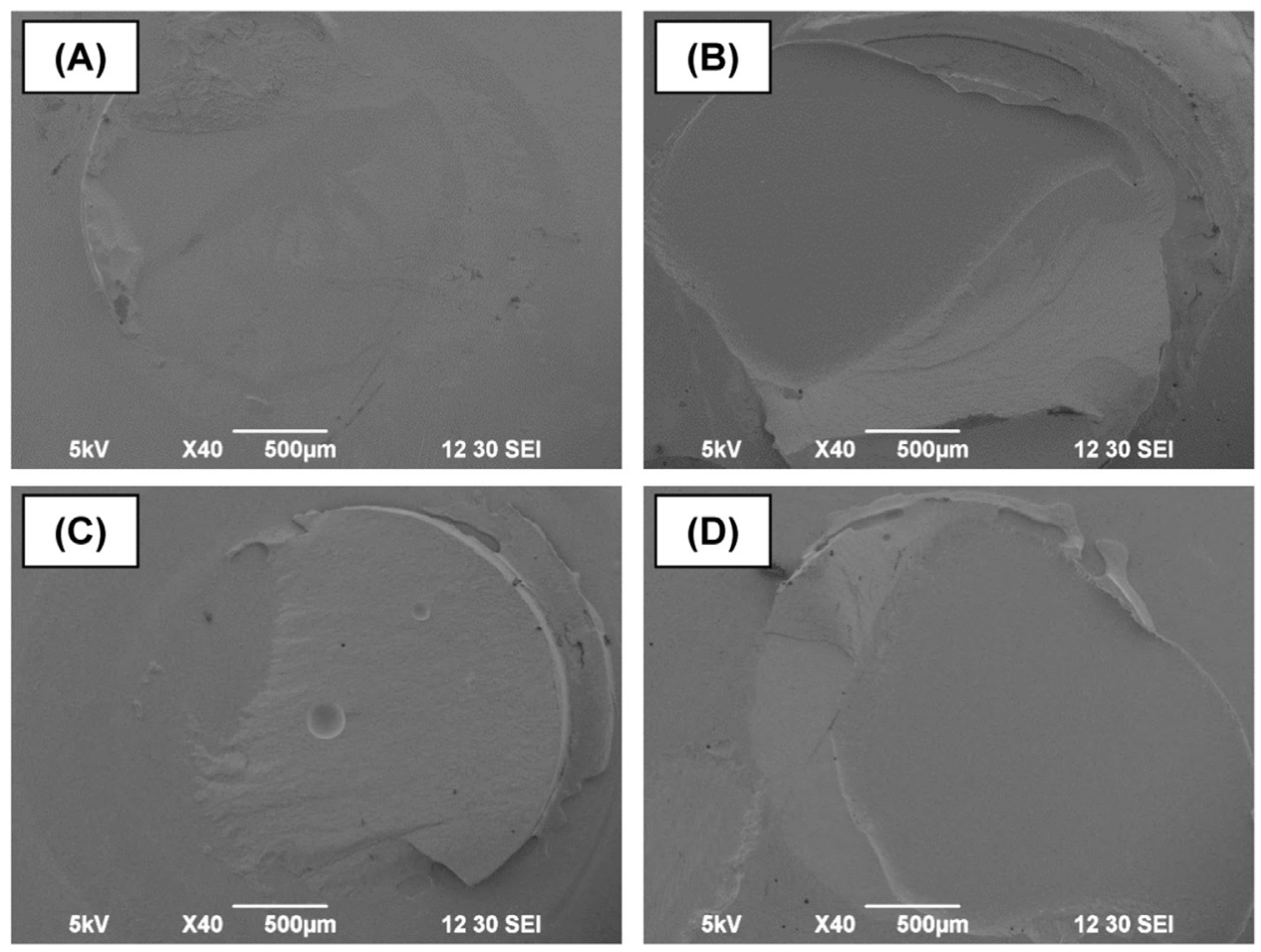

Fig. 4. SEM micrographs of the debonded FCZ surface. CJ (24 h) (A): The failure mode was classified as mixed; PS + GL (24 h) (B): The failure mode was classified as mixed. CJ (Storage/TC) (C): The failure mode was classified as mixed; PS + GL (Storage/TC) (D): The failure mode was classified as mixed.

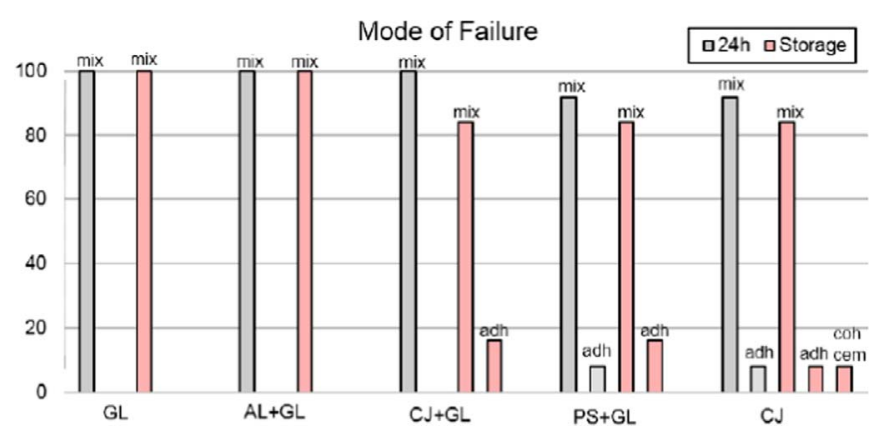

Fig. 5. Classification of the type of failure.

thickness revealed a glaze layer of $1.86 \mu \mathrm{m}$ in all groups regardless of the pretreatment type. This thickness is lower than those reported by recent studies $(6.9-10 \mu \mathrm{m})[30,31]$. One plausible explanation for such a marked difference could be due to the difference in the application methods. The present study kept the ratio of glaze paste to glaze liquid the same in all treatments and the glaze protocol included a waiting period of $2 \mathrm{~h}$ i.e., a drying time at $37^{\circ} \mathrm{C}$ before proceeding with the firing process. This step might have allowed for the removal of excess glaze liquid (including solvent present on its composition) and consequently contributed to a thin layer of glaze.

SEM analysis after scratch test revealed circular cracks in the GL group and conformal cracks in the others groups (Fig. 3). Groups, AL + GL, CJ + GL, and PS + GL, presented similar patterns where conformal cracking was observed, with clear, small areas of glaze chipping from the zirconia substrate. Classified as a cohesive failure, conformal cracking occurs as a result of the coating trying to conform to the shape of the diamond groove, resulting in open arcs away from the direction of the test [24]. The scratch test pattern results suggested that the chemical-treatment does not improve the bond strength between glaze and FCZ since all the groups (except GL group) presented the same behavior during the test. The SEM micrograph of the GL group suggests that a large amount of glaze was removed, which could result in a weak adhesion between the glaze and zirconia. It is unclear how this would impact the long-term survival of full-contour zirconia restorations treated with glaze. More studies using scratch test and glaze application associated with long-term storage are warranted.

In vitro studies $[30,32,33]$ have shown that zirconia-glazed surface could be a simple and inexpensive treatment that increases bond strength to resin cement. In the present study, the SBS results after $24 \mathrm{~h}$ (wet) and after thermocycling and water storage were statistically similar the same for the group GL and CJ, which are different from the other groups where the values decreased after aging. Currently, there is no consensus on the appropriate method necessary to evaluate the bond strength among different substrates. We have previously reported that it is important for the bonding interface to be tested at a region away from the applied mechanical stress i.e., shear, tensile, microshear and microtensile test [34].

Studies on longer exposure to the PS and the application of other resin-based cements are needed. Furthermore, future studies on the influence of thin layer glaze application on marginal adaptation of zirconia crowns should be performed. Lastly, a limitation of the proposed method is the additional number of steps included in the glaze technique, when compared to other zirconia bonding strategies using primers.

\section{Conclusions}

The results of the present study suggest no significant long-term difference among the groups when zirconia is pretreated (AL + GL, CJ + GL and PS + GL) before glaze application, when compared to the control groups without surface pretreatment (GL and CJ). The study findings suggest that FCZ surface treatment with glaze application could be considered as an alternative to traditional conditioning methods such as aluminum oxide or silica-coating since it led to similar resin bond strength and bond durability without the need for airborneparticle abrasion.

\section{Acknowledgments}

The first author (Feitosa SA) received supports (2012/02945-0) from the Sao Paulo Research Foundation (FAPESP, Brazil) and Coordination for the Improvement of Higher Education Personal (CAPES/Brazil) (18922/12-0). The authors would like to thank Ivoclar- 


\section{Vivadent for materials donation.}

\section{References}

[1] Shin YJ, Shin Y, Yi YA, Kim J, Lee IB, Cho BH, et al. Evaluation of the shear bond strength of resin cement to Y-TZP ceramic after different surface treatments. Scanning 2014;36:479-86.

[2] Feitosa SA, Corazza PH, Cesar PF, Bottino MA, Valandro LF. Pressable feldspathic inlays in premolars: effect of cementation strategy and mechanical cycling on the adhesive bond between dentin and restoration. J Adhes Dent 2014;16:147-54.

[3] Passos SP, Valandro LF, Amaral R, Ozcan M, Bottino MA, Kimpara ET. Does adhesive resin application contribute to resin bond durability on etched and silanized feldspathic ceramic? J Adhes Dent 2008;10:455-60.

[4] Kukiattrakoon B, Thammasitboon K. The effect of different etching times of acidulated phosphate fluoride gel on the shear bond strength of high-leucite ceramics bonded to composite resin. J Prosthet Dent 2007;98:17-23.

[5] Zogheib LV, Bona AD, Kimpara ET, McCabe JF. Effect of hydrofluoric acid etching duration on the roughness and flexural strength of a lithium disilicate-based glass ceramic. Braz Dent J 2011;22:45-50.

[6] Kukiattrakoon B, Thammasitboon K. Optimal acidulated phosphate fluoride gel etching time for surface treatment of feldspathic porcelain: on shear bond strength to resin composite. Eur J Dent 2012;6:63-9.

[7] Steinhauser HC, Turssi CP, Franca FM, Amaral FL, Basting RT. Micro-shear bond strength and surface micromorphology of a feldspathic ceramic treated with different cleaning methods after hydrofluoric acid etching. J Appl Oral Sci 2014;22:85-90.

[8] Ozcan M, Vallittu PK. Effect of surface conditioning methods on the bond strength of luting cement to ceramics. Dent Mater 2003;19:725-31.

[9] Bottino MA, Snellaert A, Bergoli CD, Özcan M, Bottino MC, Valandro LF. Effect of ceramic etching protocols on resin bond strength to a feldspar ceramic. Oper Dent 2015;40:40-6.

[10] Tanıș MÇ, Akay C, Karakıș D. Resin cementation of zirconia ceramics with different bonding agents. Biotechnol Biotechnol Equip 2015;29:363-7.

[11] Valandro LF, Della Bona A, Bottino MA, Neisser MP. The effect of ceramic surface treatment on bonding to densely sintered alumina ceramic. J Prosthet Dent 2005;93:253-9.

[12] de Castro HL, Corazza PH, Paes-Júnior Tde A, Della Bona A. Influence of Y-TZP ceramic treatment and different resin cements on bond strength to dentin. Dent Mater 2012;28:1191-7.

[13] Atsu SS, Kilicarslan MA, Kucukesmen HC, Aka PS. Effect of zirconium-oxide ceramic surface treatments on the bond strength to adhesive resin. J Prosthet Dent 2006;95:430-6.

[14] Melo RM, Souza R, Dursun E, Monteiro E, Valandro LF, Bottino MA. Surface treatments of zirconia to enhance bonding durability. Oper Dent 2015;40:636-43.

[15] Aboushelib MN, Kleverlaan CJ, Feilzer AJ. Selective infiltration-etching technique for a strong and durable bond of resin cements to zirconia-based materials. J Prosthet Dent 2007;98:379-88.
[16] Aboushelib MN, Feilzer AJ, Kleverlaan CJ. Bonding to zirconia using a new surface treatment. J Prosthodont 2010;19:340-6.

[17] Aboushelib MN. Evaluation of zirconia/resin bond strength and interface quality using a new technique. J Adhes Dent 2011;13:255-60.

[18] Ntala P, Chen X, Niggli J, Cattell. Development and testing of multi-phase glazes for adhesive bonding to zirconia substrates. J Dent 2010;38:773-81.

[19] Kitayama S, Nikaido T, Maruoka R, Zhu L, Ikeda M, Watanabe A, et al. Effect of an internal coating technique on tensile bond strengths of resin cements to zirconia ceramics. Dent Mater J 2009;28:446-53.

[20] Lohbauer U, Zipperle M, Rischka K, Petschelt A, Müller FA. Hydroxylation of dental zirconia surfaces: characterization and bonding potential. J Biomed Mater Res B Appl Biomater 2008;87:461-7.

[21] Zandparsa R, Talua NA, Finkelman MD, Schaus SE. An in vitro comparison of shear bond strength of zirconia to enamel using different surface treatments. J Prosthodont 2014;23:117-23.

[22] Bull SJ. Failure mode maps in the thin film scratch adhesion test. Tribology Int 1997;30:491-8.

[23] Tomastik JC, Ctvrtlik R. Nanoscratch test - a tool for evaluation of cohesive and adhesive properties of thin films and coatings. Eur Phys J Conf 2013;48:1-4.

[24] ASTM C1624-05. Standard test method for adhesion strength and mechanical failure modes of ceramic coatings by quantitative single point scratchtesting 1 C1624 - 05 (Reapproved 2010); 2010. p. 1-28.

[25] Sabrah AH, Cook NB, Luangruangrong P, et al. Full-contour Y-TZP ceramic surface roughness effect on synthetic hydroxyapatite wear. Dent Mater 2013;29:666-73.

[26] Luangruangrong P, Cook NB, Sabrah AH, et al. Influence of full-contour zirconia surface roughness on wear of glass-ceramics. J Prosthodont 2014;23:198-205.

[27] Preis V, Behr M, Hahnel S, et al. In vitro failure and fracture resistance of veneered and full-contour zirconia restorations. J Dent 2012;40:921-8.

[28] Ghazal M, Kern M. The influence of antagonistic surface roughness on the wear of human enamel and nanofilled composite resin artificial teeth. J Prosthet Dent 2009;101:342-9.

[29] Palasuk J, Platt JA, Cho SD, Levon JA, Brown DT, Hovijitra ST. Effect of surface treatments on microtensile bond strength of repaired aged silorane resin composite. Oper Dent 2013;38:91-9.

[30] Vanderlei A, Bottino MA, Valandro LF. Evaluation of resin bond strength to yttriastabilized tetragonal zirconia and framework marginal fit: comparison of different surface conditionings. Oper Dent 2014;39:50-63.

[31] Cura C, Özcan M, Isik G, Saracoglu A. Comparison of alternative adhesive cementation concepts for zirconia ceramic: glaze layer vs zirconia primer. J Adhes Dent 2012;14:75-82.

[32] Valentino TA, Borges GA, Borges LH, Platt JA, Correr-Sobrinho L. Influence of glazed zirconia on dual-cure luting agent bond strength. Oper Dent 2012;37:181-7.

[33] Everson P, Addison O, Palin WM, Burke FJ. Improved bonding of zirconia substructures to resin using a "glaze-on" technique. J Dent 2012;40:347-51.

[34] Feitosa SA, Patel D, Borges AL, Alshehri EZ, Bottino MA, Özcan M, et al. Effect of cleansing methods on saliva-contaminated zirconia - an evaluation of resin bond durability. Oper Dent 2015;40:163-71. 